Editorial

\title{
PEDIATRIC NON-ALCOHOLIC FATTY LIVER DISEASE: A GROWING PROBLEM
}

\author{
Claudia DELLA CORTE, Valerio NOBILI*
}

\author{
Hepatometabolic Department, Liver Unit \\ Bambino Gesù Children's Hospital - IRCCS \\ Rome, Italy \\ *Corresponding author: \\ nobili66@yahoo.it \\ Tel.: + 668592192 \\ Fax.: + 668593889
}

Received/Accepted: August 3, 2015

Key words: NAFLD - Treatment - Children.

Following the epidemic of pediatric obesity, non-alcoholic fatty liver disease (NAFLD) is becoming the commonest cause of chronic hepatopathy in children with exponential increase of its prevalence. The consequences of this phenomenon are the onset, also in children and adolescents, of metabolic comorbidities previously seen only in adults, with alarming effects on quality of life and life expectancy. It is estimated that non-alcoholic steatohepatitis (NASH), the advanced form of fatty liver, will represent in the near future the main indication for liver transplantation with consequent increase of organ demand and direct and indirect costs.

In a recent issue of Pediatrics Today, Fitzpatrick and Hadzic provide a concise and comprehensive overview on actual knowledge on NAFLD in pediatric setting (1). In the recent years many advances have been made in the pathogenesis/risk factors and therapeutic approach in children with NAFLD/NASH. As stated in this review, ge- netic factors now seems to play a pivotal role in pathogenesis and progression of NAFLD/ $\mathrm{NASH}$, with emerging interesting effects also on therapeutic response (1). In fact, genomewide association studies have recently identified some genetic polymorphisms in gene involved in crucial mechanisms of NAFLD development and progression that confer a greater risk of more severe, fibrotic forms. These polymorphisms was recently combined in a kit able to identify in the broad group of children with NAFLD the patients at higher risk to develop progressive and more aggressive forms (2). In the coming years, these and other novel genetic risk factors being identifies in genome wide association studies with important implications in clinical practice, improving the diagnostic protocols identifying early the most severe cases. Moreover, recent data demonstrated that genetic variants are responsible also of different therapeutic responses to some pharmacological treatment actually proposed. Particularly, it has recently reported that the presence of $148 \mathrm{M}$ allele of Patatin-like phospholipase domain containing-3 (PNPLA3) is associated with a lower response to Docosahexanoic acid (DHA) supplementation in children with NAFLD (3). This data is particularly interesting because of it opens the door for a new era of pharmacological approach to NASH, based on genetic variability. The increase of knowledge in genetic determinants of NAFLD will lead in the near future to personalize thera- 
peutic strategies, tailoring the therapeutic options on the single patients, based on its genetic and environmental risk factors.

The other interesting and evolving aspects of pediatric NAFLD are represented by therapeutic approach and clinical management (4). To date, as pointed out by the Authors in this review, do not exist guideline for the management of pediatric NAFLD (1). It is accepted that NAFLD should be now considered as the hepatic manifestation of metabolic syndrome, and, therefore, the patient with NAFLD should be evaluated for all metabolic complications that constitute the framework of metabolic syndrome. Therefore, the patients should undergo to a complete clinical and laboratory evaluation in order to explore all metabolic features related to NAFLD/NASH and metabolic syndrome. Another important aspect is that NAFLD continue to be a diagnosis of exclusion and, therefore, all possible causes of chronic steatotic hepatitis should be ruled out by appropriate tests (Fig. 1). The gold standard for diagnosis, even if invasive, expensive, and burdened by the risk of sampling error, remains liver biopsy. Recently, the Hepatology Committee of European Society of Pediatric Gastroenterology, Hepatology And Nutrition (ESPGHAN) have published a position paper for the diagnosis of NAFLD/NASH.

Based on the new knowledge in terms of risk factors and pathogenesis of NAFLD, in the past ten years, several studies have evaluated the effects of different molecules, such as insulin-sensitizers, anti-oxidants, and cytoprotective agents in the treatment of pediatric fatty liver (4). The main objective of pharmacological intervention in NAFLD is to revert liver damage and restore metabolic homeostasis. None of the available drugs is completely satisfactory and today diet and lifestyle modifications remains the cornerstone of treatment. Unfortunately, lifestyle interventions are difficult to implement with an extremely low percentage of individuals who are able to steadily lose weight and exercise regularly. For these reason several studies were conducted in adults and children in order to identify an effective strategy. In

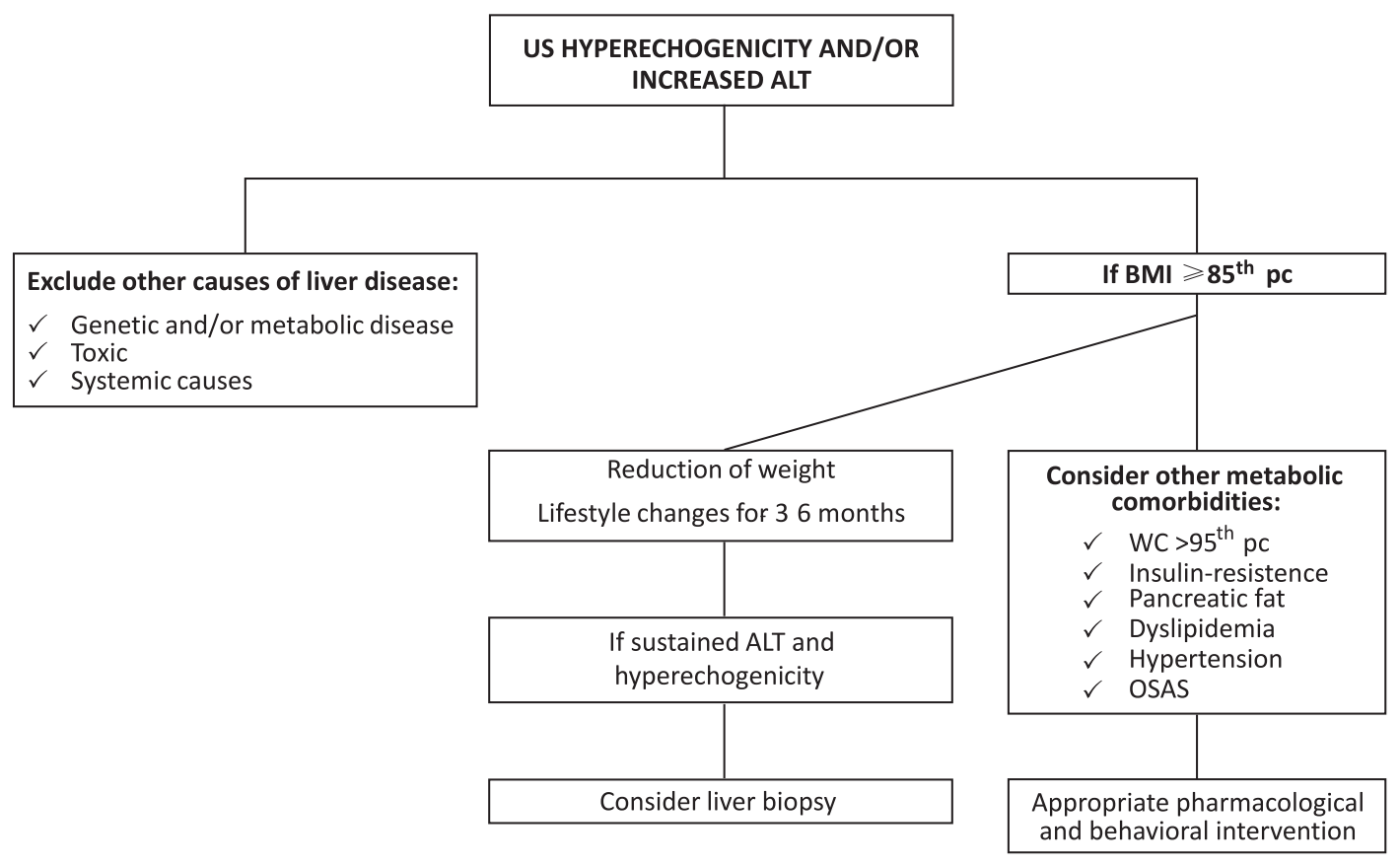

Fig. 1 Diagnostic work up for children with suspected NAFLD. 
the last few years, dietary supplementations with omega-3 fatty acids and probiotics have played a leading role in the scientific scenario of pharmacological treatment of NAFLD. Many studies have reported interesting results about these new therapeutic options. DHA, an omega-3 polyunsatured fatty acid (PUFA), showed both anti-inflammatory and insulin sensitizing properties. A randomized controlled trial on the use of DHA in pediatric NAFLD demonstrated that DHA supplementation is able to induce amelioration in insulin sensitivity index, triglycerides and ALT serum levels, steatosis at 6 months by ultrasound and at 18 months by liver biopsy. Unfortu- nately, DHA showed no effect on liver fibrosis, with amelioration of steatosis, ballooning and lobular inflammation. Based on these promising results and the optimal safety profile, DHA represents a useful option in the treatment of pediatric NAFLD (4).

Following the growing data on "gut-liver axis" in the pathogenesis of chronic hepatopathies, such as NAFLD, probiotics were tested in some animal and human studies with intriguing results. The data available for pediatric NAFLD suggested that probiotics warrant consideration as an additional beneficial therapy in these patients, demonstrating a positive effect on echographic liver steatosis, amino-

\section{Table 1 RCTs ongoing for pharmacological treatments of pediatric NAFLD}

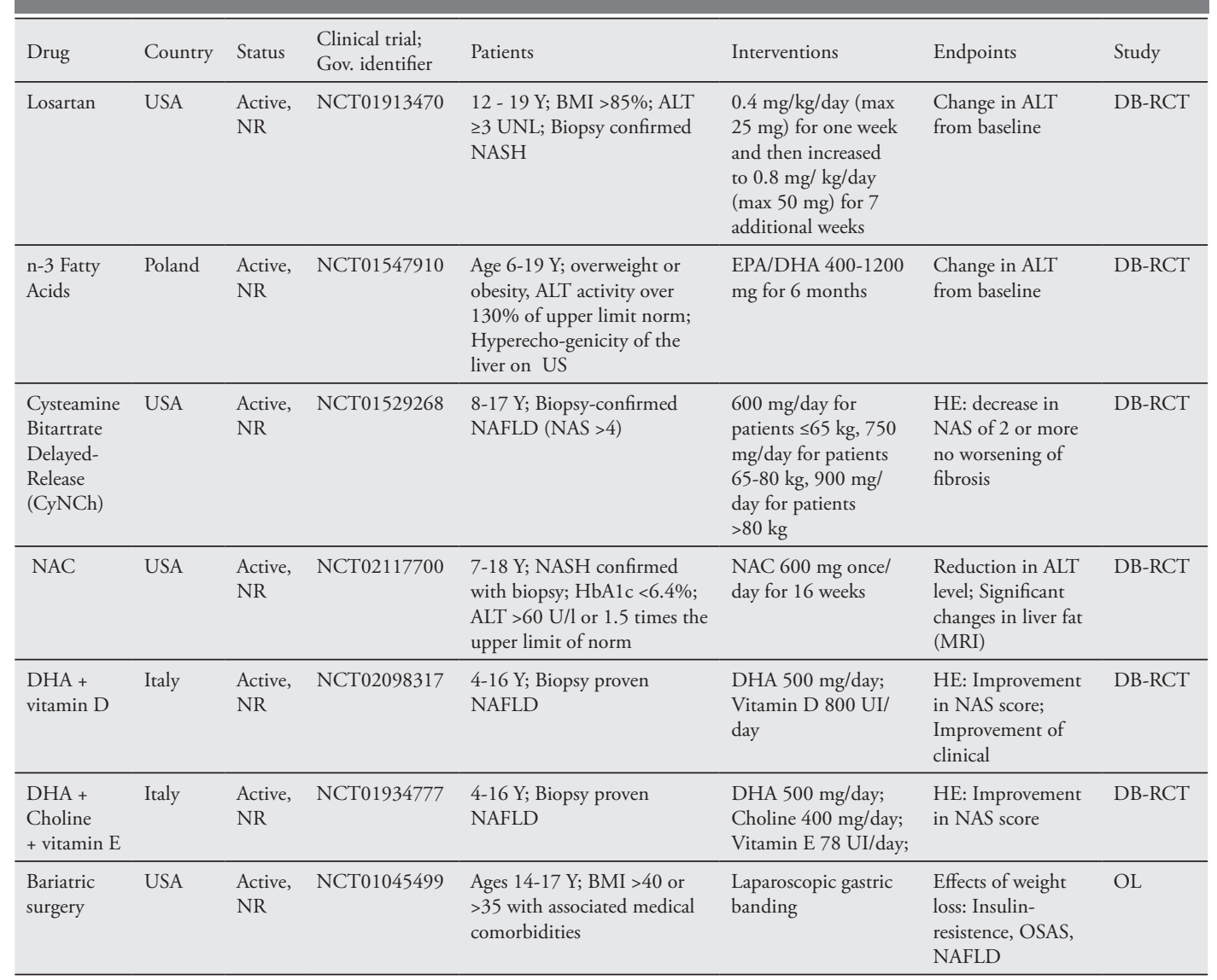

RCTs=Randomized controlled trials; NAFLD=Non-alcoholic fatty liver disease; NR=Not recruiting; NASH=Non-alcoholic steatohepatitis; $\mathrm{Y}=$ years; $\mathrm{UNL}=\mathrm{Upper}$ normal levels; DB-RCT=Double blind randomized controlled trial; DHA=Docosahexanoic acid; US=ultrasound; NAC=N-acetyl-cysteine; NAS=NAFLD Activity Score; HE=Histological endpoints; OSAS=Obstructive sleep apnoea syndrome; $\mathrm{OL}=\mathrm{Open}$ label. 
transferases serum levels and BMI; in addition, a positive effects on markers of inflammation and metabolic parameters, as cholesterol-low density lipoprotein (LDL) and insulin-resistance, were reported in adults treated with a mixture of probiotics (4).

Based on the advances in pathogenetic mechanisms of NAFLD/NASH, several molecular pathways have been identified as possible targets of novel treatment for NAFLD. Actually, some clinical randomized controlled trials are ongoing in pediatric population (Table 1) testing the efficacy of ACEinhibitors, anti-oxydants ( $\mathrm{N}$-acetyl-cysteine, cysteamine, choline and vitamin E) and anti-inflammatory molecules (vitamin D) in NAFLD/NASH (4).

Other emerging treatment modality in severely obese children with NAFLD is bariatric surgery. Recent data by a metanalasys including 15 studies showed that bariatric procedures lead to a significative improvement of liver injury in the vast majority (75\%) of treated patients, with positive effects also on fibrotic pattern. On the wave of positive results and good safety profile observed in adults population, in United Stated has been observed in 6 years (1997-2003) an increase of 5-fold in number of bariatric surgical procedures performed in adolescents. Recently, the Hepatology Committee of ESPGHAN published a societary position statement about indication of bariatric surgery in severe obese adolescents concluding that bariatric surgery should be considered a valuable therapeutic option in selected patients with BMI $>40 \mathrm{~kg} / \mathrm{mq}$ and severe comorbidities (including NASH with advanced fibrosis) or with BMI $>50 \mathrm{~kg} / \mathrm{mq}$ and mild comorbidities (5).

As previously stated, during the past decade, our understanding of pediatric NAFLD in terms of pathogenesis and risk factors has improved considerably, with a high clinical translationality in diagnosis as well as in therapy, but more advances are required to identify novel satisfactory therapeutic agents. Probably, considered the pathogenetic complexity of this disorder and its important metabolic implications, a multi-disciplinary program including behavioral intervention and multi-targeted treatments tailored on single patients, comprising pharmacological and surgical options, are required to combat the escalation and progression of this disease.

Authors' contributions: CDC and VN: Drafting the article and Revising it critically for important intellectual content.

Conflict of interest: The authors declare that they have no conflict of interest.

\section{References}

1. Fitzpatrick E, Hadzic N. Paediatric non alcoholic fatty liver disease: An emerging threat. Paediatrics Today 2015;11(1):1-9. DOI 10.5457/p2005-114.104.

2. Nobili V, Donati B, Panera N, Vongsakulyanon A, Alisi A, Dallapiccola B, et al. A 4-polymorphism risk score predicts steatohepatitis in children with nonalcoholic fatty liver disease. J Pediatr Gastroenterol Nutr. 2014;58:632-6.

3. Nobili V, Bedogni G, Donati B, Alisi A, Valenti L. The I148M variant of PNPLA3 reduces the response to docosahexaenoic acid in children with non-alcoholic fatty liver disease. J Med Food. 2013; 16(10):957-60.

4. Della Corte C, Liccardo D, Ferrari F, Alisi A, Nobili V. Current pharmacotherapy for treating pediatric nonalcoholic fatty liver disease. Expert Opin Pharmacother. 2014; 15(17):2501-11.

5. Nobili V, Vajro P, Dezsofi A, Fischler B, Hadzic N, Jahnel J, et al. Indications and limitations of bariatric intervention in severely obese children and adolescents with and without nonalcoholic steatohepatitis: ESPGHAN Hepatology Committee Position Statement. J Pediatr Gastroenterol Nutr. 2015; 60(4):550-61. 